\title{
Fast Acquisition for Transmitted Reference Ultra- Wideband Systems with Channelized Receiver
}

\author{
Lei Feng, Won Namgoong \\ Department of Electrical Engineering \\ University of Southern California \\ leifeng@usc.edu,namgoong@usc.edu
}

\begin{abstract}
The acquisition performance of the channelized receiver for ultra-wideband (UWB) transmitted reference (TR) system is presented. Instead of sampling the received UWB signal with a single ADC as in a conventional fullband receiver, the channelized receiver digitizes with multiple slow ADCs, each of which samples a partial band of the UWB signal. In this paper, we show that the frequency channelized receiver naturally leads to fast acquisition. In the channelized receiver, the reduced bandwidth in each subband widens the correlation peak, allowing the search increment to be correspondingly increased. Consequently, the search space in the channelized receiver is reduced, resulting in significantly faster acquisition time than in a fullband receiver.
\end{abstract}

\section{INTRODUCTION}

Transmitted reference (TR) system is attractive in many ultra-wideband (UWB) applications because it simplifies the channel estimation process, which is a key challenge in UWB systems [1][2]. In a UWB TR system, detection can be performed by simply correlating consecutive received pulses since channel is assumed to be constant for the two-pulse period and the pulse interval is large enough to prevent inter-pulse interference.

Since designing a single ADC to operate at the signal Nyquist rate is difficult, parallel ADC architectures with each ADC operating at a fraction of the Nyquist rate need to be employed. To sample at a fraction of the Nyquist rate, a frequency channelized receiver based on hybrid filter banks (i.e., continuous-time analysis filters and discrete-time synthesis filters) has been proposed in [3]. Among the advantages of the frequency channelized receivers compared to the more conventional time channelized (i.e., time-interleaved ADC) receivers are the ease of designing the sample/hold circuitries, greater robustness to jitter/phase noise, and reduced ADC dynamic range requirements. The detection structure of the channelized receiver in UWB TR systems is described in [4][5].

This work was supported in part by the Army Research Office under contract number DAAD19-01-1-0477 and National Science Foundation under contract number ECS-0134629.
This paper deals with coarse acquisition in UWB TR system. Since the transmitter and receiver clocks are not synchronized, the receiver must first perform coarse acquisition to estimate the pulse arrival time and the pulse repetition period before detection. The ultra-narrow UWB pulse makes the correlation very sensitive to time offset, requiring small search steps to achieve acquisition. The search space is also large because of the low duty cycle of the TR signal. As a result, fast acquisition is an active research topic [6][7][8].

In this paper, we show that the frequency channelized receiver naturally leads to fast acquisition. Coarse acquisition in a TR system is achieved by performing a two-dimensional search for the pulse arrival time and the interval between consecutive pulses. The acquisition hypothesis variable is obtained by correlating between consecutive pulses in each subband, squaring to remove the phase uncertainty, then summing. For comparison, a fullband receiver, which is equivalent to a onesubband channelized receiver, is also considered. In the channelized receiver, the reduced bandwidth in each subband widens the correlation peak, allowing the search increment to be correspondingly increased. Consequently, the search space in the channelized receiver is reduced, resulting in a significantly faster acquisition time than that of a fullband receiver.

The paper is organized as following. The TR signals of a channelized receiver is described in Section II. The coarse acquisition performance is derived in Section III. Conclusions are drawn in Section IV.

\section{TRANSMITTED REFERENCE SIGNALS}

To satisfy the FCC spectral mask, we use the 5th-derivative of the Gaussian pulse [9]

$$
p_{t r}(t)=A_{c}\left(-\frac{t^{5}}{\sqrt{2 \pi} \sigma^{5}}+\frac{t^{3}}{\sqrt{2 \pi} \sigma^{3}}-\frac{t}{\sqrt{2 \pi} \sigma}\right) e^{-\frac{t^{2}}{2 \sigma^{2}}}
$$

where $A_{c}$ is a constant and $\sigma$ of $51 \mathrm{ps}$ fills the FCC spectral mask in the $3.1-10.6 \mathrm{GHz}$ band. Since designing a receiver to support the entire UWB band may be difficult, we subsequently assume a more practical pulse with a bandwidth of $1.5 \mathrm{GHz}(3.1-4.6 \mathrm{GHz})$, which is obtained by filtering the pulse response in (1) with a fourth order Chebyshev bandpass filter. The resulting pulse waveform is shown in Fig.1. 


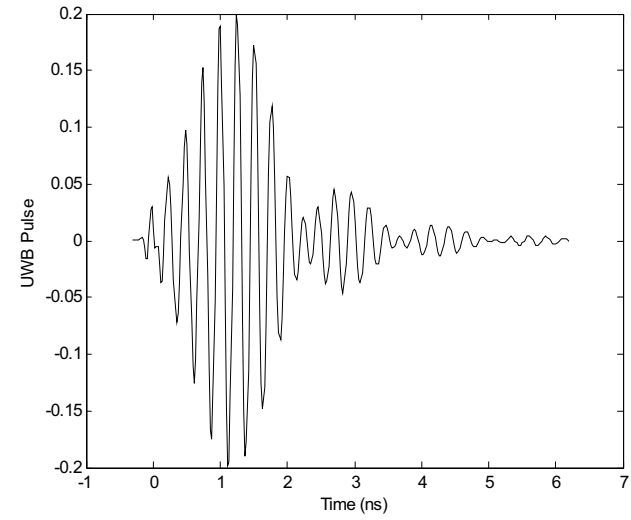

Fig.1 The transmitted pulse with $1.5 \mathrm{GHz}$ bandwidth

After passing through the multipath channel, the received pulse is $s(t)$. In each transmission block, the first several pulses are reference pulses and the remaining are data pulses. In this paper, we do not distinguish between the reference and data pulses for acquisition analysis. To simplify the problem, we assume that the pulses are transmitted in fixed time intervals and that the interval is sufficiently large to prevent inter-pulse interference between consecutive pulses. The channel is assumed to be constant during a transmission block. A block of received signal is

$$
r(t)=\sum_{k=0}^{N_{d}-1} a_{k} s\left(t-k T-N_{r} T\right)+n(t)
$$

where $a_{k}$ is the $k$ th transmitted antipodal symbol, $n(t)$ is the additive noise, and $T$ is the frame period (pulse repetition period). The channel estimation can be achieved by averaging the reference pulses. The estimated pulse is then correlated with the other frame signals to detect the data. The short transmitted pulse duration indicates that the detection is very sensitive to timing offset.

The channelized receiver is an efficient method to digitize UWB signals since directly sampling the UWB signal with a single ADC is difficult due to ADC sampling rate limitations. An $M$ subband channelizer is shown in Fig.2. The received UWB signal $r(t)$ is downconverted by a set of equally spaced mixers at frequencies $f_{0}, f_{1}, \ldots f_{M-1}$, filtered by the lowpass filter $H(\omega)$, then sampled by ADCs operating at a fraction of the effective sampling frequency. The effective sampling frequency $\left(T_{S}\right)$ is set to exceed the signal Nyquist rate. The subband ADC sampling frequency is $f_{s u b}=1 / M T_{s}$. Let $h(t)$ be the impulse response of the lowpass filter. In the $k$ th pulse and $m$ th subband, the subband sample $x_{k, m}[i]=x_{k, m}\left(i M T_{s}\right)$, $(m=0,1, \ldots, M-1$ and $i=0,1, \ldots)$, is

$$
\begin{aligned}
& x_{k, m}[i]= \\
& \left.\left\{\left[a_{k} s(t-k T)+n(t)\right] e^{-j 2 \pi f_{m} t}\right\} \otimes h(t)\right|_{t=k(T+\varepsilon)+i M T_{s}} \\
& \quad=a_{k} e^{-j 2 \pi f_{m} k T_{s_{m}}\left(k \varepsilon+i M T_{s}\right)+n_{m, k}[i]}
\end{aligned}
$$

$T+\varepsilon$ is the receiver's estimate of the pulse repetition period $T$. The filtered subband pulse and noise are

$$
\begin{gathered}
s_{m}(t)=\left[s(t) \otimes\left(h(t) e^{j 2 \pi f_{m} t}\right)\right] e^{-j 2 \pi f_{m} t} \\
n_{m, k}[i]=\left.n(t) e^{-j \omega_{m} t} \otimes h(t)\right|_{t=k(T+\varepsilon)+i M T_{s}}
\end{gathered}
$$

The channelized receiver for UWB TR systems has been shown to perform better than a fullband receiver, which is assumed to sample the UWB signal with a single ADC [4].

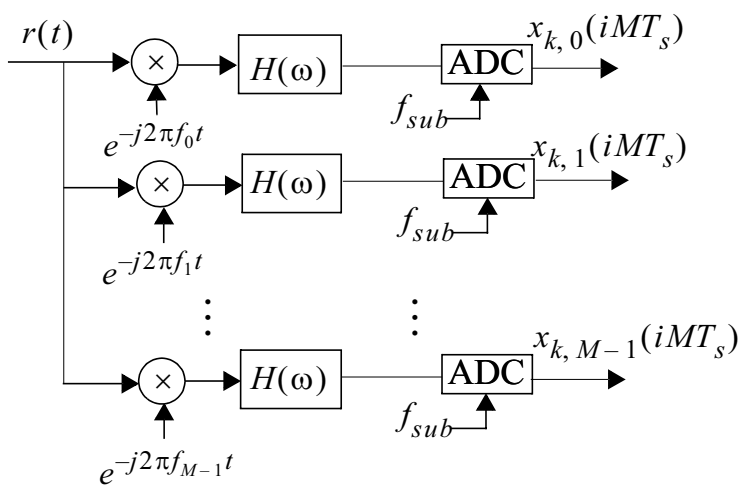

Fig.2 An $M$ subband UWB Channelizer

For comparison purposes, the received signal in a fullband receiver is described as follows. The fullband ADC samples at the effective sampling rate of $1 / T_{s}$. Denoting the mixer frequency as $f_{f}$, the $i^{\prime}$ th sample of the $k$ th pulse is

$$
\begin{aligned}
& x_{k}\left[i^{\prime}\right]= \\
& \left.\left(a_{k} s(t-k T)+n(t)\right) e^{-j 2 \pi f_{f} t}\right|_{t=k(T+\varepsilon)+i^{\prime} T_{s}} \\
& =a_{k} e^{-j 2 \pi f_{f} k T} s_{f u l l}\left(k \varepsilon+i^{\prime} T_{s}\right)+n_{f u l l, k}\left[i^{\prime}\right]
\end{aligned}
$$

The filtered fullband noise is

$$
\begin{gathered}
s_{f u l l}(t)=s(t) e^{-j 2 \pi f_{f} t} \\
n_{f u l l, k}\left[i^{\prime}\right]=\left.n(t) e^{-j 2 \pi f_{f} t}\right|_{t=k(T+\varepsilon)+i^{\prime} T_{s}}
\end{gathered}
$$

\section{COARSE ACQUISITION}

Since the transmitter and receiver clocks are not synchronized, the receiver must first perform coarse acquisition at the sampling rate to estimate the pulse arrival time $\tau_{0}$ and the pulse repetition period $T$ before detection. To reduce the "noise cross noise" term, a time window is applied to each pulse in order to capture only the high signal-to-noise ratio part. The acquisition parameters $\left(\tau_{0}\right.$ and $\left.T\right)$ are shown in Fig.3. 
$\tau_{0}$ is searched by sliding the two windows by a number of sampling periods within the pulse repetition period $T$ while keeping their interval constant. $\tau_{0}$ takes the values $i M T_{s}\left(i T_{s}\right.$ for a fullband receiver), $i=0,1, \ldots$. Due to the incommensurate rate between $T$ and $T_{s}, \tau_{0}$ usually does not repeat after exceeding $T$. However, with sufficiently high sampling rate, we assume $\tau_{0}$ repeats for ease of analysis. For each tentative value of $\tau_{0}$, the pulse repetition period $(T)$ is searched from all possible time offsets by a step equal to the sampling period. The acquisition procedure of $T$ is to change the timing offset between two windows in the range corresponding to the clock uncertainty. The total number of search states is the multiplication of the numbers of two possible values.

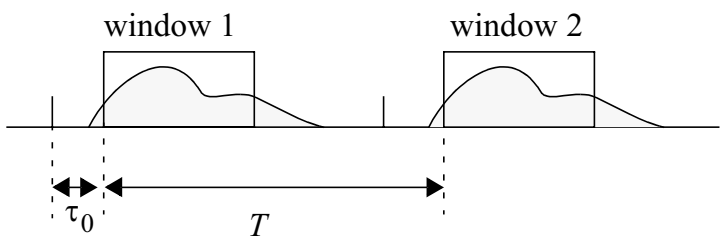

Fig.3 Acquisition parameters

In a full-band receiver, the coarse acquisition is achieved by first correlating the conjugate of the windowed $k$ th pulse with the windowed $(k+1)$ st pulse. The cross-correlation value is squared to remove any phase ambiguity. If the resulting hypothesis variable exceeds a certain threshold, the receiver enters the fine synchronization mode. If the resulting value is below the threshold, the receiver changes the tentative values of $\tau_{0}$ and/or $T$ before continuing the search process.

The coarse acquisition process in the channelized receiver is similar to that in the full-band receiver. In each subband, the conjugate of the windowed $k$ th pulse is correlated with the windowed $(k+1)$ st pulse. The cross-correlation value in each subband is squared then summed. The estimates of both $\tau_{0}$ and $T$ are varied during the acquisition process. Coarse acquisition is completed when the resulting hypothesis variable exceeds a certain threshold. Compared to the full-band receiver, the squaring and summing operation in each subband reduces the probability of detection $P_{D}$ for a given $P_{F}$, which is the false alarm probability when signal is absent in both pulses. Despite this degradation, the overall coarse acquisition time improves, because the frequency channelization process reduces the number of search bins. Since the reduced bandwidth in each subband channel widens the correlation peak by approximately the number of subbands $M$, the search increment can be increased by $M$, resulting in a reduction of the search space by a factor of $M$. Assuming no degradation in the detection probability $P_{D}$, the acquisition time reduces by almost $M$ times. An example of the correlation for a fullband and 3-subband frequency channelized receiver is plotted in Fig. 4 as a function of the offset time assuming CM1 channel and no additive noise. Note that in addition to the widening of the correlation peak, multiple sidelobes emerge in the frequency channelized receiver, resulting in increased $P_{F}$.

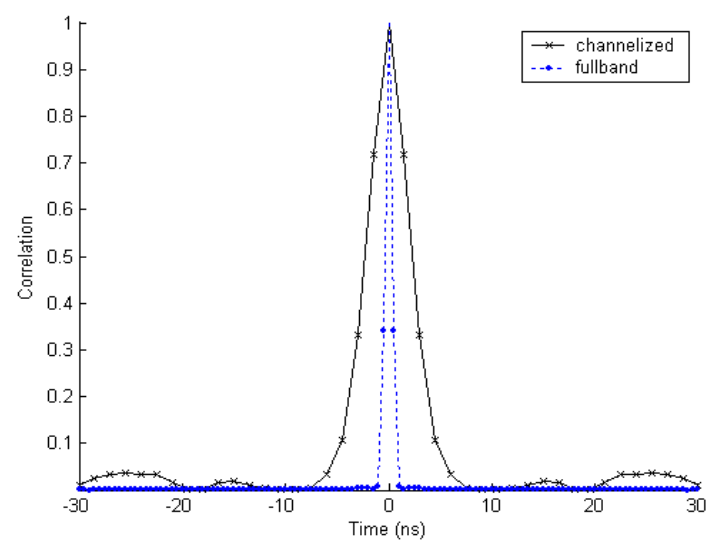

Fig.4 The correlation values of a fullband signal and 3subband channelized signals with respect to timing offset between two windows

In this section, the acquisition performance of both the full-band and channelized receivers are analyzed. Each search state corresponds to a pair of $\tau_{0}$ and $T$ values. Serial-search algorithm is considered, that is, only one state is checked for a pulse period $T$. If successful acquisition is not reached at current state $l$, state $l+1$ is searched in the next period.

\section{III.1 Hypothesis testing in a full-band receiver}

The hypothesis testing is performed to decide whether correct acquisition is achieved when searching over all the states. The search states are labeled by $l=0,1, \ldots, L_{1}-1$. The $l=0$ state corresponds to the correct acquisition state. For simplicity, we assume all pulses are unmodulated. At the $l$ th state, the samples in the windowed $k$ th pulse given in (6) can be represented as a $N_{w}$ dimension vector

$$
\mathbf{x}_{f u l l, l, k}=e^{j \theta_{f u l l, k}\left(\mathbf{s}_{f u l l, l, k}+\mathbf{n}_{f u l l, l, k}\right)}
$$

where $\theta_{f u l l, k}=-2 \pi f_{f} k T$ is the $k$ th pulse initial phase, $\mathbf{s}_{f u l l, l, k}$ is the signal vector and $\mathbf{n}_{f u l l, l, k}$ is the complex noise vector with covariance matrix $N_{0} \mathbf{I}$ with $\mathbf{I}$ being an identity matrix. $\theta_{f u l l, k}$ is time varying due to transmitter and receiver clock uncertainty. Correlating with the windowed $(k+1)$ st pulse, the resulting signal is

$$
\begin{aligned}
Y_{f u l l, l}= & \mathbf{x}_{f u l l, l, k}^{H} \mathbf{x}_{f u l l, l, k+1} \\
= & e^{j\left(\theta_{f u l l, k+1}-\theta_{f u l l, k}\right)}\left(\mathbf{s}_{f u l l, l, k}^{H} \mathbf{s}_{f u l l, l, k+1}\right. \\
& +\mathbf{s}_{f u l l, l, k}^{H} \mathbf{n}_{f u l l, l, k+1}+\mathbf{n}_{f u l l, l, k}^{H} \mathbf{s}_{f u l l, l, k+1} \\
& \left.+\mathbf{n}_{\text {full }, l, k}^{H} \mathbf{n}_{f u l l, l, k+1}\right)
\end{aligned}
$$

where the superscript $H$ stands for conjugate transpose. The first term in the parenthesis on the right hand side of (10) is the 
signal component and the remaining terms are the noise components. The second and third terms are "signal cross noise" terms and are zero mean Gaussian noise. The fourth term, which is the "noise cross noise" term, is also approximately Gaussian by the Central Limit Theorem, since the noise in different pulses is independent. The magnitude of the mean of $Y_{\text {full, }, l}$ is

$$
E_{f u l l, l}=\left|\mathbf{s}_{\text {full, } l, k}^{H} \mathbf{s}_{f u l l, l, k+1}\right|
$$

and the variance of all the noise is

$$
\sigma_{\text {full, } l}^{2}=\left|\mathbf{s}_{\text {full }, l, k}\right|^{2} \sigma^{2}+\left|\mathbf{s}_{\text {full }, l, k+1}\right|^{2} \sigma^{2}+N_{w} \sigma^{4}
$$

To remove the phase ambiguity, the hypothesis variable is obtained by squaring the correlated signal

$$
J_{f u l l, l}=\left|Y_{f u l l, l}\right|^{2}
$$

The hypothesis variable $J_{f u l l, l}$ is then compared to a threshold to determine whether the signal is present. $J_{f u l l, l}$ has a central/noncentral chi-square distribution with two degrees of freedom depending on whether the signal is present or not. Denoting the threshold as $\gamma$, the probability that the hypothesis variable exceeds $\gamma$ is [10]

$$
P_{f u l l, l}=\int_{\gamma / \sigma_{\text {full, } l}^{2}}^{\infty} e^{-\left(x+\mu_{f u l l, l}\right)} I_{0}\left(2 \sqrt{x \mu_{f u l l, l}}\right) d x
$$

where $\mu_{f u l l, l}=E_{f u l l, l}^{2} / \sigma_{f u l l, l}^{2}$ and $I_{0}$ is zeroth order modified Bessel function of the first kind. Since the correct acquisition state is $0, P_{f u l l, 0}$ is the detection probability and $P_{f u l l, l}$ $\left(l=1, \ldots, L_{1}-1\right)$ are false alarm probabilities.

\section{III.2 Hypothesis testing in a channelized receiver}

The search states for a channelized receiver are labeled by $l=0,1, \ldots, L_{2}-1$. The $l=0$ state corresponds to the correct acquisition state. In the channelized receiver, $N_{w} / M$ samples are collected at a rate of $1 /\left(M T_{s}\right)$ for each pulse. The search increment step for time offset $T$ is $M T_{s}$ instead of $T_{s}$ as in the fullband receiver. Because of the increased search increment step, the number of channelized receiver search states is reduced by $M$ compared to a fullband receiver, that is, $L_{1}=M L_{2}$.

The ADC samples in the $m$ th subband of the windowed $k$ th pulse is represented in vector form as

$$
\mathbf{x}_{m, l, k}=e^{j \theta_{m, k}}\left(\mathbf{s}_{m, l, k}+\mathbf{n}_{m, l, k}\right)
$$

where $\mathbf{s}_{m, l, k}$ is the signal vector, $\mathbf{n}_{m, l, k}$ is the noise vector and $\theta_{m, k}$ is the $m$ th subband mixer phase at the beginning of the $k$ th pulse. After correlating with the windowed $(k+1)$ st pulse in the corresponding subband, squaring and then summing all the subband correlation signals, the hypothesis variable of the channelized receiver is

$$
\begin{aligned}
& J_{c h, l}= \\
& M-1 \\
& \sum_{m=0}\left|\left(\mathbf{s}_{m, l, k}+\mathbf{n}_{m, l, k}\right)^{H}\left(\mathbf{s}_{m, l,(k+1)}+\mathbf{n}_{m, l,(k+1)}\right)\right|^{2}
\end{aligned}
$$

As in the full-band receiver, the hypothesis variable $J_{c h, l}$ is compared with a threshold to determine whether the signal is present. Assuming that the noise among the subbands is uncorrelated, $J_{c h, l}$ becomes the summation of $M$ independent and chi-square distributed random variables. The probability density function (PDF) of $J_{c h, l}$ is the convolution of $M$ chi-square PDFs, each of which corresponds to the squared correlation signal in a subband. The false alarm and detection probabilities, $P_{c h, l}\left(l=1, \ldots, L_{2}-1\right)$ and $P_{c h, 0}$, can then be readily obtained by integrating the PDF of $J_{c h, l}$.

\section{III.3 Acquisition performance comparison}

Following similar steps as in [10], the single-dwell serialsearch mean acquisition time of a fullband receiver in terms of $T$ is

$$
\begin{aligned}
& T_{\text {acq, full }}=\frac{2 L_{1}-\left(L_{1}-1\right) P_{\text {full }, 0}}{2 P_{f u l l} L_{1}^{0}-1} \\
& +\frac{\kappa\left(1-P_{f u l l, 0}\right) \sum_{l=1} P_{f u l l, l}}{P_{f u l l, 0}}+\frac{\sum_{l=1} l P_{f u l l, l}}{L_{1}}
\end{aligned}
$$

where $\kappa$ is false alarm penalty. In the channelized receiver, the mean acquisition time is similar to (17) except that $P_{\text {full, } l}$ ( $\left.l=0, \ldots, L_{2}-1\right)$ and $L_{1}$ are replaced by $P_{c h, l}$ and $L_{2}$, respectively.

$$
\begin{aligned}
T_{a c q, c h}= & \frac{2 L_{2}-\left(L_{2}-1\right) P_{c h, 0}}{2 P_{c h, 0_{L_{2}-1}}} \\
& +\frac{\kappa\left(1-P_{c h, 0}\right) \sum_{l=1} P_{c h, l}}{P_{c h, 0}}+\frac{L_{2}-1}{l=1} \sum_{c h, l}
\end{aligned}
$$

To understand the operating characteristic of the decision device in both receivers, the detection probability $P_{D}$, and the false alarm probability when signal is absent in both pulses $P_{F}$ are plotted in Fig.5. The received $E_{b} / N_{0}=5 \mathrm{~dB}$ or $15 \mathrm{~dB}$. The channelized receiver has three subbands. For a given $P_{F}$, the $P_{D}$ of the full-band receiver is higher than that of the channelized receiver, because of the loss due to the squaring and summing of the subband signals. 


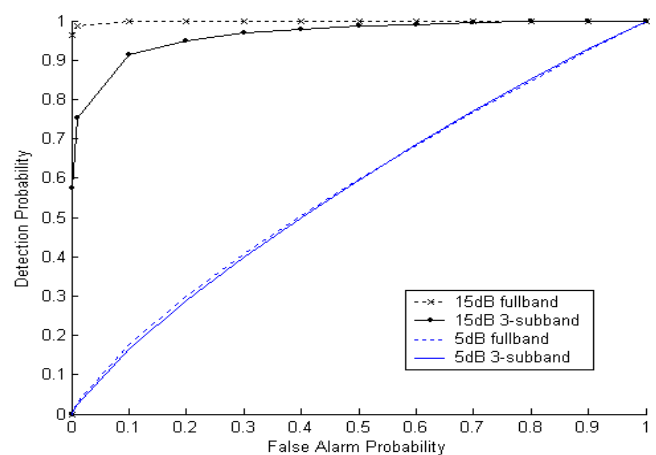

Fig.5 The operating characteristic of the decision device

Although $P_{D}$ is lower for a given $P_{F}$, an important advantage of the frequency channelized receiver is that the number of bins to search is reduced by approximately $M$ as explained earlier. The net result of these two contradictory effects is that the ratio of the mean acquisition time between the channelized and the fullband receivers depends on the number of subbands and $E_{b} / N_{0}$. Using (17) and (18), the acquisition time ratios at $E_{b} / N_{0}$ of $5 \mathrm{~dB}$ and $15 \mathrm{~dB}$ are plotted in Fig.6 as a function of the number of subbands for a CM1 channel. To compute the acquisition time, we assume that the penalty associated with a false alarm is five time units, i.e., $\kappa=5$. The mean acquisition time is shorter in a channelized receiver than in a fullband receiver due to the reduced search space. As the number of subbands increases, however, the reduction in mean acquisition time suffers from diminishing returns because of the increase in false alarm probability.

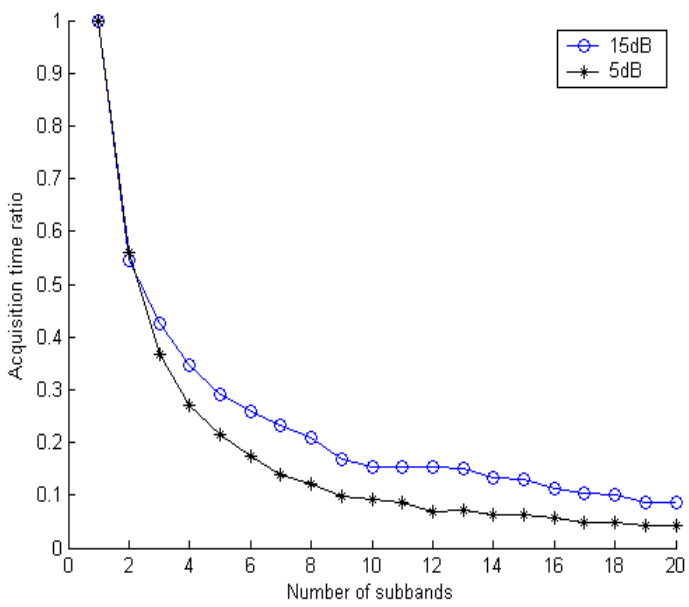

Fig.6 Acquisition time ratio between channelized receivers and a fullband receiver

\section{CONCLUSIONS}

In this paper, the acquisition performances of both the fullband and channelized receivers are analyzed. A closed form equation for acquisition time is derived given $P_{D}$ and $P_{F}$. Although the channelized receiver suffers from combining loss compared to a fullband receiver, the reduced bandwidth in each subband widens the correlation peak, resulting in a reduced search space. As a result, the acquistion time of the frequency channelized receiver is significantly faster than in a fullband receiver.

\section{REFERENCE}

[1] R. Hoctor and H. Tomlison, "An Overview of DelayHopped, Transmitted-Reference RF Communications," GE Research and Development Center, Tech. Rep. 2001CRD198, 2002

[2] Y. Chao and R. A. Scholtz, "Optimal and suboptimal receivers for ultra-wideband transmitted reference systems," GLOBECOM '03, vol. 2, pp759 - 763, Dec. 2003

[3] W. Namgoong, "A Channelized Digital Ultra-Wideband Receiver,” IEEE Trans. Wireless Comm., vol. 2, pp502510, May 2003

[4] L. Feng and W. Namgoong, "A Oversampled Channelized UWB Receiver," International Workshop on UWBST \& IWUWBS 2004, pp:410 - 414, May 2004

[5] L. Feng and W. Namgoong, "Oversampled Channelized Receiver for Transmitted Reference UWB System in the Presence of Narrowband Interference," IEEE Workshop on Signal Processing Systems (SIPS) 2004, pp:48 - 52, Oct. 2004

[6] E.A. Homier and R.A. Scholtz, "Rapid Acquisition of Ultra-wideband Signals in the Dense Multipath Channel," Ultra Wideband Systems and Technologies (UWBST) 2002, pp105 - 109, May 2002

[7] R. Blazquez, P. Newaskar and A. Chandrakasan, "Coarse Acquisition for Ultra Wideband Digital Receivers," ICASSP 2003, vol. 4, pp IV - 137-140, April 2003

[8] L. Reggiani and G. M. Maggio, "Rapid search algorithms for code acquisition in UWB impulse radio communications," IEEE Journal on Selected Areas in Communications, vol. 23, Issue 5, pp 898 - 908, May 2005

[9] ,H. Kim D. Park and Y. Joo, “All-digital low-power CMOS pulse generator for UWB system," IEE Electronics Letters, vol. 40, NO.24, Nov. 2004

[10] K. SH. Zigangirov, "Theory of Code Division Multiple Access Communication,” John Wiley \& Sons, Inc. 2004 